\title{
Optimal Control Problems with Maximum Functional
}

\author{
Ping $\mathrm{Lu}^{*}$ \\ Iowa State University, Ames, Iowa 50011 \\ and \\ Nguyen X. Vinh $\dagger$ \\ University of Michigan, Ann Arbor, Michigan 48109
}

\begin{abstract}
This paper considers the optimal control problem with a performance index that includes a maximum functional. Necessary conditions are derived that are more general than those of previous work, some of which is shown to be reducible and, therefore, included in the results found here. An illustrative example demonstrates that necessary conditions are satisfied by a unique solution of the problem. The problem of threat avoidance for aircraft is formulated as the optimal control problem considered here. Numerical results for a subsonic glider are presented.
\end{abstract}

\section{Introduction}

$\mathrm{N}$ this pap problem:

$$
\begin{gathered}
\min \left\{\max _{t_{0} \leq t \leq t_{f}} F(x(t))+\phi\left(x\left(t_{f}\right), t_{f}\right)\right\} \\
\dot{x}(t)=f(x(t), u(t)) \\
u(t) \in U, \quad \forall t \in\left(t_{0}, t_{f}\right) \\
x\left(t_{0}\right)=x_{0} \\
S\left(x\left(t_{f}\right)\right)=0
\end{gathered}
$$

where, in the performance index of Eq. (1), $F(x)$ and $\phi(x, t)$ are two scalar functions with continuous partial derivatives, $x \in X \subset R^{n}$ is the state, $X$ is the state space, $u(t)$ is an $m$-dimensional piecewise continuous control function, $U \subset R^{m}$ is a control set, and $S\left(x\left(t_{f}\right)\right)=\left\{s_{1}\left(x\left(t_{f}\right)\right), s_{2}\left(x\left(t_{f}\right)\right), \ldots, s_{r}\left(x\left(t_{f}\right)\right)\right\}^{T}$ represents a smooth terminal manifold of dimension $n-r$, $r \leq n$. The final time $t_{f}$ is implicitly defined by Eq. (5), or prescribed, and $f(x, u): R^{n} \times R^{m} \rightarrow R^{n}$ is assumed to be $C^{1}$. Here, without loss of generality, we only consider the autonomous system, because a nonautonomous system can always be transformed into an autonomous system by an additional variable $x_{n+1}=t$. Similarly, we do not include an integral in the cost function because it can also be easily transformed into a function of the final state $x\left(t_{f}\right)$, and, therefore, included in $\phi$.

The maximum functional term $\max F(x(t))$ in Eq. (1), as named by Bellman, ${ }^{1}$ makes the problem a nonclassical optimal control problem. If $F(x)=0$ in the cost function, the problem reduces to a problem of Mayer, for which well-developed theory exists; if $\phi=0$, the problem is called the Chebyshev minimax optimal control problem.

Optimal control problems of this type arise in various areas of science and engineering. Many efforts have been devoted to the solutions of this problem and the minimax problem. ${ }^{1-16}$ In Ref: 1 , a geometrical approach to the problem is given. A transform technique is introduced in Ref. 2 to solve an auxil-

Received March 26, 1990; revision received Oct. 10, 1990; accepted for publication Oct. 10, 1990. Copyright (C) 1991 by the American Institute of Aeronautics and Astronautics, Inc. All rights reserved.

*Assistant Professor, Department of Aerospace Engineering and Engineering Mechanics. Member AIAA.

$\dagger$ Professor, Department of Aerospace Engineering. Member AIAA. iary problem, the solution of which can be arbitrarily close to the original problem. Although these techniques are enlightening, they are hardly applicable to problems with even only moderate complexity. Reference 3 gives an existence theorem to the problem. Reference 4 presents a set of abstract necessary conditions that are general but rather difficult to apply. Reference 5 investigates the problem when the order is higher than one, and a set of practical necessary conditions is obtained. In recent years, emerging problems of this type in the aerospace trajectory optimization have prompted renewed interest in seeking their solutions. Solving a transformed optimal control problem, Refs. 6-9 contain accurate numerical solutions to minimax optimal control problems arising from aeroassisted orbital transfer. References 10-12 apply the technique successfully in the treatment of strategies for flight in a wind shear (both takeoff and abort landing). Reference 13 treats the problem with scalar control, and discusses the numerical procedure.

Compared with the successful numerical solutions to this type of problem, a study of the necessary conditions that are practical and applicable to a broader class of problems appears to lag behind. Yet the necessary conditions can not only provide guidance for numerical computation, but can also help in understanding the problem better and can sometimes reveal important features of the problem before the numerical solution is obtained. ${ }^{14}$ The objective of this paper is twofold: First, we use a transform technique to obtain necessary conditions for the problem, and comparison of these necessary conditions with some previous results is conducted. Next, to demonstrate the application, we formulate the aircraft threat avoidance problem within the frame of this type of problem. Numerical results are presented for the optimal trajectories of an aeroglider.

\section{Necessary Conditions}

Suppose that $x^{*}(t), u^{*}(t)\left(t_{0} \leq t \leq t_{f}\right)$ is an optimal solution pair to the problem in this section and the next, where $x *(t)$ is absolutely continuous and $\boldsymbol{u}^{*}(t)$ is piecewise continuous. Also suppose that $F(x)$ is at least $C^{q}$, where $q \geq 1$, as defined in Definition 1 in this section. We need the following definitions in order to present the necessary conditions.

\section{Definition 1}

Let $\left[t_{i}, t_{i}^{\prime}\right] \subset\left(t_{0}, t_{f}\right), t_{i} \leq t_{i}^{\prime}, t_{i}^{\prime}<t_{i+1}, i=1, \ldots, k, k \geq 1$, be all the intervals in $\left(t_{0}, t_{f}\right)$, such that $\forall \tau \in\left[t_{i}, t_{i}^{\prime}\right]$, $F\left(x^{*}(\tau)\right)=\max F\left(x^{*}(t)\right), t \in\left[t_{0}, t_{f}\right]$. If $t_{i}=t_{i}^{\prime}, F\left(x^{*}(t)\right)$ is said to have an isolated maximum at $t_{i}$. If $t_{i}<t_{i}^{\prime}, F\left(x^{*}(t)\right)$ is said to have a flat maximum in $\left(t_{i}, t_{i}^{\prime}\right)$. 


\section{Remark}

Note that we exclude the case when $F\left(x^{*}(t)\right)$ attains its maximum at $t_{f}$ or $t_{0}$, because if $F\left(x^{*}\left(t_{f}\right)\right)$ or $\left\{F\left(x^{*}\left(t_{0}\right)\right)\right\}$ $=\max F\left(x^{*}(t)\right)$, the performance index can be expressed as $\boldsymbol{F}\left(\boldsymbol{x}\left(t_{f}\right)\right)$ or $\left\{\boldsymbol{F}\left(\boldsymbol{x}\left(t_{0}\right)\right)\right\}+\phi\left(\boldsymbol{x}\left(t_{f}\right), t_{f}\right)$. The problem simply reduces to a problem of Mayer.

\section{Definition 2}

If $F^{(q)}\left(x^{*}(t), u^{*}(t)\right)$, the $q$ th order derivative of $F\left(x^{*}(t)\right)$ is the first derivative for all $q \geq 1$ to satisfy the following condition:

$$
F_{u}^{(q)}\left(x^{*}(t), u^{*}(t)\right) \neq 0
$$

so that for all $t$ in any of the finite intervals $\left(t_{i}, t_{i}^{\prime}\right), t_{i}<t_{i}^{\prime}$, defined in Definition $1, F(x)$ is said to be of order $q$.

\section{Remark}

If $F(x *(t))$ attains its maximum only at some isolated points in $\left(t_{0}, t_{f}\right)$, the order of $F(x(t))$ is immaterial, as we shall see. Hence, we do not define the order of $F(x)$ in this case.

\section{Definition 3}

Let $F(x(t))$ be of order one, $\left(t_{i}, t_{i}^{\prime}\right) \subset\left(t_{0}, t_{f}\right)$ be a finite interval in which $F\left(x^{*}(t)\right)$ attains flat maximum, and $\forall \tau \in\left(t_{i}, t_{i}^{\prime}\right)$, $\omega_{i}\left(x^{*}(\tau)\right) \subset U$ be defined as the set of all $u \in U$, such that

$$
\begin{gathered}
\dot{F}(x *(\tau), u)=0 \\
\dot{F}_{u}\left(x^{*}(\tau), u\right) \neq 0
\end{gathered}
$$

\section{Remark}

Under the assumption that $x^{*}(t)$ and $u^{*}(t)$ exist and $F(x(t))$ is of order one, $\omega_{i}\left(x^{*}(\tau)\right)$ is not empty because $u=u^{*}(\tau)$, $\tau \in\left(t_{i}, t_{i}^{\prime}\right)$ satisfies Eqs. (7) and (8). In the case where the control $u$ is scalar, or $\dot{F}$ only contains one component of vector control $u, \omega_{i}\left(x^{*}(\tau)\right)$ is solved directly from Eq. (7), provided that the resultant $\omega_{i}\left(x^{*}(\tau)\right)$ is in $U \subset R$.

After this preparation, let is consider the following optimal control problem equivalent to the problem of Eqs. (1-5):

$$
\begin{gathered}
\min \left\{x_{n+1}\left(t_{f}\right)+\phi\left(x\left(t_{f}\right), t_{f}\right)\right\} \\
\dot{x}(t)=f(x(t), u(t)) \\
\dot{x}_{n+1}=f_{n+1}(x(t), u(t)) \triangleq 0 \\
u(t) \in U, \quad \forall t \in\left(t_{0}, t_{f}\right) \\
F(x)-x_{n+1} \leq 0 \\
x\left(t_{0}\right)=x_{0} \\
x_{n+1}\left(t_{0}\right)=\text { free } \\
S\left(x\left(t_{f}\right)\right)=0
\end{gathered}
$$

where the introduced auxiliary variable $x_{n+1}$ is actually a constant. Apparently any optimal solution of Eqs. (1-5) will be an optimal solution of Eqs. (9-15), with $x_{n+1}=\max F\left(x^{*}(t)\right)$, $t_{0} \leq t \leq t_{f}$, and vice versa. Equations (9-15) represent a problem of Mayer with inequality state constraint for which extensive theoretical results are available. We shall primarily utilize the work by Pontryagin et al. ${ }^{17}$ to derive our necessary conditions.

First, we consider the situation when $F(x *(t))$ only has isolated maximum.

\section{Necessary Condition I}

Suppose $F\left(x^{*}(t)\right)$ attains its maximum only at some isolated points $t_{i} \in\left(t_{0}, t_{f}\right), i=1, \ldots, k, k \geq 1$. Then there exists a nonzero piecewise absolutely continuous vector function $\left(p^{T}(t), p_{n+1}(t)\right) \triangleq\left(p_{1}(t), \ldots, p_{n}(t), p_{n+1}(t)\right)$, called the adjoint state, such that, for the Hamiltonian defined by

$$
H(x, p, u)=p^{T} f(x, u)+p_{n+1} f_{n+1}=p^{T} f(x, u)
$$

the following must be satisfied for almost all $t \in\left(t_{0}, t_{f}\right)$ :

$$
\begin{gathered}
\dot{x}^{*}=H_{p}\left(x^{*}, p, u^{*}\right) \\
\dot{p}=-H x\left(x^{*}, p, u^{*}\right) \\
\ddot{p}_{n+1}=-H_{x_{n+1}}\left(x^{*}, p, u^{*}\right)=0 \\
H\left(x^{*}, p, u^{*}\right)=\sup _{u \in U} H\left(x^{*}, p, u\right)=C
\end{gathered}
$$

where $C$ is a constant. At $t_{1}, t_{2}, \ldots, t_{k}$, the following jump conditions hold:

$$
\begin{gathered}
p\left(t_{i}^{+}\right)=p\left(t_{i}^{-}\right)+\mu_{i} F_{x}\left(x^{*}\left(t_{i}\right)\right) \\
p_{n+1}\left(t_{i}^{+}\right)=p_{n+1}\left(t_{i}^{-}\right)-\mu_{i} \\
\mu_{i} \geq 0, \quad i=1, \ldots, k
\end{gathered}
$$

The transversality conditions at $t_{0}$ and $t_{f}$ are

$$
\begin{gathered}
p_{n+1}\left(t_{0}\right)=0 \\
p\left(t_{f}\right)=\phi_{x}\left(x^{*}\left(t_{f}\right),\left(t_{f}\right)\right) \psi_{0}+S_{x}^{T}\left(x^{*}\left(t_{f}\right)\right) \boldsymbol{\beta} \\
p_{n+1}\left(t_{f}\right)=\psi_{0} \\
H\left(t_{f}\right)=-\phi_{t}\left(\boldsymbol{x} *\left(t_{f}\right), t_{f}\right) \psi_{0} \\
\psi_{0} \leq 0, \quad \boldsymbol{\beta}=\left(\beta_{1}, \ldots, \beta_{n-r}\right)^{T}
\end{gathered}
$$

Let us outline the proof. Notice that, for the problem (9-16), when $F\left(x^{*}(\tau)\right)=\max F\left(x^{*}(t)\right), t_{0} \leq t \leq t_{f}$, the inequality state constraint [Eq. (13)] becomes active at $\tau$, since $x_{n+1}$ $=\max F\left(x^{*}(t)\right), t_{0} \leq t \leq t_{f}$. The necessary conditions for a general problem with inequality constraint $g(x(t)) \leq 0$ are given in Ref. 17, with the assumption that for those $t \in\left(t_{0}, t_{f}\right)$, such that $g(x *(t))=0$,

$$
\dot{g}_{u}\left(x^{*}(t), u^{*}(t)\right) \neq 0
$$

But in Ref. 16 it is shown that this condition is not needed if $g\left(x^{*}(t)\right)=0$ only at some isolated points, which in our case corresponds to $F\left(x *\left(t_{i}\right)\right)=\max F\left(x^{*}(t)\right)=x_{n+1}, t_{0} \leq t \leq t_{f}$, only at some isolated $t_{i}$. Therefore, by following Theorems 1 and 3 in Ref. 17 and Theorem 4 in Ref. 16, these necessary conditions are in order.

In the transversality conditions (26-28), we shall concentrate on the nondegenerate case only, namely, $\psi_{0} \neq 0$. Without loss of generality, we take $\psi_{0}=-1$. From Eqs. (20) and (23), $p_{n+1}$ is piecewise constant. From Eqs. (23), (25), and (27), with $\psi_{0}=-1$, we arrive at

$$
\sum_{i=1}^{k} \mu_{i}=1
$$

Rearranging the jump conditions for $p$, we have

$$
\begin{gathered}
p\left(t_{i}^{+}\right)=p\left(t_{i}^{-}\right)+\mu_{i} F_{x}\left(x *\left(t_{i}\right)\right) \\
\sum_{i=1}^{k} \mu_{i}=1 \\
\mu_{i} \geq 0, \quad i=1, \ldots, k
\end{gathered}
$$

Note that all equations related to $p_{n+1}$ are only used to obtain 
Eq. (31) and that $p_{n+1}$ will not appear in the actual application of the necessary conditions.

Next, we consider the cases where $F\left(x^{*}(t)\right)$ has flat maximum.

\section{Necessary Condition II}

Assumption 1: If $F\left(x^{*}(t)\right)$ has flat maximum in $\left(t_{0}, t_{f}\right)$, $F(x(t))$ is assumed to be of order one, and $u^{*}(t)$ is not on the boundary of the control set $U$ for all $t$ in these finite intervals where the flat maximum of $F\left(x^{*}(t)\right)$ occurs.

Suppose that $F\left(x^{*}(t)\right)$ attains its maximum $k$ times in $\left(t_{0}, t_{f}\right), k \geq 1$, including flat maximum in some finite intervals $\left(t_{j}, t_{j}^{\prime}\right), j=1, \ldots, l, l \leq k$. Outside the intervals $\left(t_{j}, t_{j}^{\prime}\right)$, Necessary Condition I applies. Inside the intervals $\left(t_{j}, t_{j}^{\prime}\right)$, the following must hold under our stated assumption:

$$
\begin{gathered}
\dot{x}^{*}=H_{p}\left(x^{*}, p, u^{*}\right) \\
\ddot{p}=-H_{x}\left(x^{*}, p, u^{*}\right)+\nu_{j}(t) \dot{F}_{x}\left(x^{*}, u^{*}\right) \\
\dot{p}_{n+1}=-H_{x_{n+1}}\left(x^{*}, p, u^{*}\right)+\nu_{j}(t) \dot{F}_{x_{n+1}}\left(x^{*}, u^{*}\right)=0 \\
H\left(x^{*}, p, u^{*}\right)=\sup _{u \in \omega_{j}\left(x^{*}(t)\right)} H\left(x^{*}, p, u^{*}\right)=C
\end{gathered}
$$

where for $t \in\left(t_{j}, t_{j}^{\prime}\right), \nu_{j}(t)$ is a piecewise continuous function satisfying

$$
H_{u}\left(x^{*}, p, u^{*}\right)=\nu_{j}(t) \dot{F}_{u}\left(x^{*}, u^{*}\right)
$$

Note that $\omega_{j}\left(x^{*}(t)\right)$ is defined by Definition 3, mentioned earlier. At $t_{j}, j=1, \ldots, l$, the jump conditions hold:

$$
\begin{gathered}
\boldsymbol{p}\left(t_{j}^{+}\right)=\boldsymbol{p}\left(t_{j}^{-}\right)+\mu_{j} F_{x}\left(\boldsymbol{x}^{*}\left(t_{j}\right)\right) \\
\sum_{i=1}^{k} \mu_{i}=1
\end{gathered}
$$

Again, the proof is straightforward: By following Theorems 22 and 24 in Ref. 17, which deal with general optimal control problems with inequality state constraint $g(x) \leq 0$, we obtain the previously defined necessary conditions readily, if we identify $g\left(x_{1}, \ldots, x_{n}, x_{n+1}\right)=F(x)-x_{n+1}$.

Note that, according to Ref. 17, Assumption 1 can be removed with some modification in the results. We adopt it for the simplicity of the presentation.

It should be noted that although Eq. (39) is identical to Eq. (31), Eq. (32) is not necessarily true for the indices $j$ corresponding to a flat maximum.

\section{Necessary Condition III}

When $F(x(t))$ is of order higher than one and has flat maximum, if the control is scalar, a set of necessary conditions may also be derived by using the technique in Ref. 18 of treating a higher order inequality state constraint. We list the result here.

Suppose $F(x(t))$ is of order $q>1$ and has flat maximum over some finite intervals $\left(t_{j}, t_{j}^{\prime}\right), j=1, \ldots, l, l \leq k$, where $k \geq 1$ again is the number of times $F\left(x^{*}\right)$ attains its maximum. Outside $\left(t_{j}, t_{j}^{\prime}\right)$ Necessary Condition I applies. Inside $\left(t_{j}, t_{j}^{\prime}\right)$, we have

$$
\dot{p}=-H_{x}+\left(F_{u}^{(q)}\right)^{-1} F_{x}^{(q)}\left(f_{u}\right)^{T} p
$$

The jump conditions are

$$
\begin{gathered}
\boldsymbol{p}^{+}\left(t_{j}\right)=\boldsymbol{p}^{-}\left(t_{j}\right)+\mu_{j} F_{x}+N_{x} \boldsymbol{\alpha}^{j} \\
\sum_{j=1}^{k} \mu_{j}=1
\end{gathered}
$$

where

$$
N_{x}=\left(\dot{F}_{x}, \ddot{F}_{x}, \ldots, F_{x}^{(q-1)}\right)
$$

and $\left(\alpha^{j}\right)^{T}=\left(\alpha_{1}^{j}, \ldots, \alpha_{q-1}^{j}\right)^{T}$ is a multiplier vector.
Although the transform Eq. (13) was suggested in Ref. 19 in 1965 , it is surprising to find that for more than two decades very little work has been done in this direction, despite the fact that interest in seeking treatments for the problem with minimax functional has remained strong over the years and various other approaches have been studied. In the next section, we will demonstrate that a representative of previous work can be simplified and actually is relevant to our results. Some remarks are in order:

1) The unknown multiplier $\mu_{i}$ in Eqs. (30), (38), and (41) poses no extra difficulty when $F\left(x^{*}(t)\right)$ attains its maximum only once (isolated or flat), which in many cases is true, because $\mu_{1}=1$ when $k=1$.

2) The Hamiltonian $H(\boldsymbol{x}, \boldsymbol{p}, \dot{u})$ is said to be regular if it admits a unique maximizing $\boldsymbol{u}^{*}$ for given $\boldsymbol{x}$ and $p$. If $H$ is regular, it can be shown that the optimal control $u^{*}(t)$ should be continuous across the maximum point, despite the discontinuities in the adjoint states, due to the jump conditions.

3) The type of maximum that $F\left(x^{*}(t)\right)$ has, i.e., isolated or flat, and the number by which the maximum is attained must be determined before applying the necessary conditions. In some cases, the judgment may be made on the type of maximum based on the condition that the continuity of $u^{*}$ and the jump conditions should not be contradictory if the Hamiltonian is regular. Unlike the ordinary optimal control problem with inequality state constraint, where the inequality may or may not become active, the jump conditions will be applied at least once in the current problem.

4) These necessary conditions reduce to the necessary conditions for the Chebyshev minimax problem given in Refs. 14-16 when $\phi=0$.

\section{Comparison with Previous Work}

Previous work in the literature that is closest to ours is in Ref. 5, in which a restricted version of the problem is investigated. The problem is transformed into an optimal control problem with intermediate point constraint within the frame of the classical calculus of variations. The following assumptions are imposed.

\section{Assumption 2}

1) $F(x *(t)), t_{0} \leq t \leq t_{f}$, has a unique isolated maximum at $t_{1} \in\left(t_{0}, t_{f}\right)$, and $F\left(x^{*}(t)\right)$ is unimodal.

2) The order of $F(x *(t))$ is greater than one. This assures that $\dot{F}\left(x^{*}\left(t_{1}\right)\right)=0$ regardless of the continuity of $u^{*}(t)$ at $t_{1}$.

3) $u^{*}(t), \forall t \in\left(t_{0}, t_{f}\right)$, is an interior of the control set $U$.

With these assumptions, the problem is converted into the following:

$$
\begin{gathered}
\min J=\min \left\{F\left(x\left(t_{1}\right)\right)+\phi\left(x\left(t_{f}\right), t_{f}\right)+\int_{t_{0}}^{t_{\overline{1}}}\left(\boldsymbol{p}^{T} \dot{x}-H\right) \mathrm{d} t\right. \\
\left.+\int_{t_{1}^{+}}^{t_{f}}\left(p^{T} \dot{x}-H\right) \mathrm{d} t\right\} \\
\dot{x}(t)=\boldsymbol{f}(\boldsymbol{x}(t), u(t)) \\
\dot{F}\left(\boldsymbol{x}\left(t_{1}\right)\right)=0 \\
x\left(t_{0}\right)=x_{0} \\
S\left(x\left(t_{f}\right)\right)=0
\end{gathered}
$$

where $H=p^{T} f(x, u)$. Equation (46) is treated as an intermediate point constraint. By adjoining the constraint (46) to $J$, the multiplier rule is applied to take the first-order variation of $J$ and set $\delta J=0$. Finally, a set of necessary conditions is obtained, which is basically the same as Eqs. (18), (19), and (21), except that the jump condition is

$$
p\left(t_{1}^{+}\right)=p\left(t_{1}^{-}\right)+F_{x}\left(x^{*}\left(t_{1}\right)\right)+\lambda \dot{F}_{x}\left(x^{*}\left(t_{1}\right)\right)
$$


where $\lambda$ in Eq. (49) is a constant multiplier. When the maximum of $F\left(x^{*}\right)$ is unique $(k=1)$, as required by Assumption 2 in this section, our jump conditions (30-32) give

$$
p\left(t_{1}^{+}\right)=\boldsymbol{p}\left(t_{1}^{-}\right)+F_{x}\left(x^{*}\left(t_{1}\right)\right)
$$

Therefore, the difference is between Eqs. (49) and (50). However, in the Appendix we show that Eq. (49) should be reduced to Eq. (50), as asserted by the following proposition.

\section{Proposition 1}

The multiplier $\lambda$ in jump condition (49) is always zero; thus, Eq. (49) is identical to Eq. (50).

Reference 5 also considers a flat maximum case when $F$ is of order higher than one. The necessary conditions determined are essentially the same as the previous Necessary Condition III, when restricted to $k=1$.

In summary, since our results do not require Assumption 2 in this section, more general cases can be treated, including cases where multiple maxima occur and $F(x)$ is of order one. Many physical problems do not meet Assumption 2. For instance, the heating rate and deceleration of an atmospheric transfer vehicle are of order one. The minimization of their peak values can be conveniently handled using the results in Sec. II.

A more recent work involving the necessary conditions is Ref. 13, where the discussion is restricted to the case of scalar control and the maximum of $F\left(x^{*}\right)$ is attained only once. As today's control systems become more advanced, the need for extending the treatment to a multicontrol case is obvious. The previously stated Necessary Conditions I and II are not restricted to scalar control, but Necessary Condition III is limited to $q>1$. This inconvenience is inherited from the maximum principle. ${ }^{17}$

\section{Illustrative Example}

Let us consider the problem

$$
\begin{gathered}
\min \left\{\max _{0 \leq t \leq t_{f}} \alpha\left[x_{1}(t)+x_{2}(t)\right]+(1-\alpha) t_{f}\right\} \\
\dot{x}_{1}=x_{2} \\
\dot{x}_{2}=u \\
|u| \leq 1 \\
x_{1}(0)=x_{2}(0)=2 \\
x_{1}\left(t_{f}\right)=x_{2}\left(t_{f}\right)=0
\end{gathered}
$$

where $0 \leq \alpha \leq 1$ places relative weightings on the two objectives. As required by the necessary conditions in Sec. II, there exist adjoint states $p_{1}(t)$ and $p_{2}(t)$ for the Hamiltonian

$$
H=p_{1} x_{2}+p_{2} u
$$

Let us assume that $F$ has an isolated maximum. The adjoint states satisfy Eq. (19), as

$$
\begin{aligned}
& \dot{p}_{1}=-H_{x_{1}}=0 \\
& \dot{p}_{2}=-H_{x_{2}}=p_{1}
\end{aligned}
$$

They are integrable

$$
p_{1}=c_{1}^{-}, \quad p_{2}=-c_{1}^{-} t+c_{2}^{-}
$$

where the superscript $\left(^{-}\right)$denotes the values before the jump conditions are applied. The Hamiltonian is a constant throughout $\left(0, t_{f}\right)$. Through Eq. (28), we have

$$
H=\frac{\partial \phi\left(x^{*}\left(t_{f}\right), t_{f}\right)}{\partial t_{f}}=1-\alpha
$$

Suppose that $F$ achieves its maximum at $t_{1} \in\left(0, t_{f}\right)$. The jump conditions $(30-32)$ require that

$$
c_{1}^{-}=c_{1}^{-}+\alpha, \quad-c_{1}^{+} t_{1}+c_{2}^{+}=-c_{1}^{-} t_{1}+c_{2}^{-}+\alpha
$$

where the superscript $\left({ }^{+}\right)$denotes the values after the jump.

By the maximum condition (21), we assume that the control takes the form

$$
u(t)=\operatorname{sign}\left[p_{2}(t)\right]= \begin{cases}-1 ; & t \in\left[0, t_{2}\right] \\ 1 ; & t \in\left(t_{2}, t_{f}\right]\end{cases}
$$

Using Eq. (63) to integrate the state equations (52) and (53) with the boundary conditions (55) and (56), $t_{2}$ and $t_{f}$ can be determined. Then, $t_{1}$ is solved. The values are

$$
\begin{gathered}
t_{1}=1, \quad t_{2}=4, \quad t_{f}=6 \\
\max _{0 \leq t \leq t_{f}} F\left[x_{1}(t), x_{2}(t)\right]=x_{1}(1)+x_{2}(1)=4.5
\end{gathered}
$$

The adjoint states are solved by using Eqs. (61-64) and the condition $p_{2}\left(t_{2}\right)=0$. Finally,

$$
\begin{aligned}
& p_{1}(t)= \begin{cases}-(1+\alpha) / 2 ; & t \in[0,1) \\
-(1-\alpha) / 2 ; & t \in[1,6]\end{cases} \\
& p_{2}(t)= \begin{cases}(1+\alpha) t / 2-2 ; & t \in[0,1) \\
(1-\alpha) t / 2-2(1-\alpha) ; & t \in[1,6]\end{cases}
\end{aligned}
$$

It is straightforward to verify that all the necessary conditions are satisfied by these controls, states, and adjoint states.

Although solutions satisfying the necessary conditions are not necessarily optimal, and no sufficient conditions for this type of problem are readily available, in this particular example we can easily argue that the control given by Eq. (63), with $t_{2}=4$, is indeed optimal for the cost function (51) for any $0 \leq \alpha \leq 1$. First, we recognize that it is the time optimal control; $t_{f}=6$ is the minimum time needed to transfer the system from the initial position (55) to the origin. Second, since $\dot{F}=x_{2}+u$, we see that $\dot{F}>0$ for an initial period of time for any admissible $u$, because of the initial condition $x_{2}(0)=2$ and the control effort limit $|u| \leq 1$. That is, $F$ is increasing from $F(0)=4$. Also notice that $F=\int_{0}^{t} u \mathrm{~d} t+u+2$. By applying $u=-1$ in that initial period, as Eq. (63) suggests, $\dot{F}$ is kept at the smallest value. Any other admissible $u \neq-1$ will yield larger $\dot{F}$, and it will take longer before $\dot{F}$ can turn negative. Therefore, the maximum value of $F$ given in Eq. (65) must be the smallest that $F$ can ever attain. In conclusion, $u(t)$, defined by Eq. (63), is optimal for each performance index $t_{f}$ and $\max \left(x_{1}+x_{2}\right)$; it must be optimal for the composite performance index (51).

It is well known that the time optimal control is unique for a linear system..$^{17}$ Hence, the previously stated arguments assert that the optimal control obtained is also unique for the cost [Eq. (51)] because any other controls, at best, can only yield the same smallest maximum of $F$, but certainly will take longer $t_{f}$.

An interesting observation is that if we are to solve the Pareto optimal control problem with two components of the vector objective function being $t_{f}$ and $\max \left(x_{1}+x_{2}\right)$, these arguments also establish that the Pareto optimal control set contains a unique point, given by Eq. (63).

Let us only consider the minimax problem by setting $\alpha=1$ in Eq. (51), as

$$
\min \left[\max _{0 \leq t \leq t_{f}}\left(x_{1}+x_{2}\right)\right]
$$

The system is subject to the same constraints defined by Eqs. (51-56), with $t_{f}$ free. As pointed out by Remark 4 at the end of Sec. II, the necessary conditions for the minimax 
problem are obtained if $\phi$ is set to zero in the necessary conditions derived in Sec. II, so that Eqs. (57-60) and Eq. (62) remain valid, and Eq. (61) becomes

$$
H=0
$$

Note that $u(t)$ from Eq. (63), with $t_{2}=4$, is also optimal for the minimax problem, as we argued. But the adjoint states by Eq. (66) are now

$$
p_{1}(t)=\left\{\begin{array}{ll}
-1 ; & t \in[0,1) \\
0 ; & t \in[1,6]
\end{array}, \quad p_{2}(t)= \begin{cases}t-2 ; & t \in[0,1) \\
0 ; & t \in[1,6]\end{cases}\right.
$$

In contrast to Eq. (66), $p_{1}$ and $p_{2}$ are both zero after $t_{1}=1$. The maximum condition (21) no longer provides information to determine the optimal control. A "singular" case appears. The explanation for this is that there exist an infinite number of optimal controls after $t_{1}$. In fact, let $0<t \leq 2$. Defining

$$
t_{2}=2+\Delta t, \quad t_{3}=\frac{4+2 \Delta t}{\Delta t}, \quad t_{f}=t_{3}+\Delta t
$$

the following control is also optimal:

$$
u= \begin{cases}-1 ; & t \in\left[0, t_{2}\right) \\ 0 ; & t \in\left[t_{2}, t_{3}\right) \\ 1 ; & t \in\left[t_{3}, t_{f}\right]\end{cases}
$$

The corresponding adjoint states are

$$
p_{\mathrm{I}}(t)=\left\{\begin{array}{ll}
-1 ; & t \in[0,1) \\
0 ; & t \in\left[1, t_{f}\right]
\end{array}, \quad p_{2}= \begin{cases}t-2 ; & t \in[0,1) \\
0 ; & t \in\left[1, t_{f}\right]\end{cases}\right.
$$

It is again easy to verify that all the necessary conditions for the minimax problem are satisfied. The maximum of $F$ is attained at $t_{1}=1$ :

$$
F\left(t_{1}\right)=x_{1}(1)+x_{2}(1)=4.5
$$

When $\Delta t=2$, Eqs. (71) and (63) give the same control. Figure 1 presents the variations of $F$ corresponding to $\Delta t=1$ and $\Delta t=2$. Figure 2 shows the trajectories in the phase plane. We note that Eq. (71) by no means exhausts all possible combinations of the optimal controls to the minimax problem. One can construct many other optimal controls. All the optimal controls, however, must be equal to -1 in the interval $(0,1)$. To admit these many optimal controls in $\left(1, t_{f}\right]$ which must satisfy the necessary conditions, in particular, the maximum condition [Eq. (21)], $p_{2}(t)$ can only be zero in $\left(1, t_{f}\right.$ ]. A vanishing $p_{2}(t)$ leads to $p_{1}(t)=0$ because of the adjoint state equation (59).

The structure of the solution to the minimax problem is not a total surprise, in view of the free terminal time. The only difference between the necessary conditions for the problem with composite cost $(51)$ and the minimax problem in this example is that $H=1-\alpha \neq 0$ [Eq. (61)] and $H=0$ [Eq. (68)], but that is enough for admitting vastly different solutions.

\section{Problem of Threat Avoidance}

The problem of determining the optimal threat avoidance trajectory of an aircraft has been discussed in Ref. 20. The objective is to find the trajectory minimizing a cost function that consists of the exposure of the aircraft to threats and other quantity, such as fuel consumption. In Ref. 20, the risk cost associated with a threat is modeled by

$$
J_{r}=\int_{t_{0}}^{t_{f}} \frac{C_{r}}{d^{2}} \mathrm{~d} t
$$

where $d$ is the distance from the threat and $C_{r}$ is a cost factor.

If the threat environment is not time-dependent and only the closeness to the threat constitutes the risk, another natural alternative of characterizing the risk index may be the use of some functions, such as exponential $C_{1} \exp \left[-C_{2} d(t)\right], C_{1}$, $C_{2}>0$, provided that the risk is considered the same from any

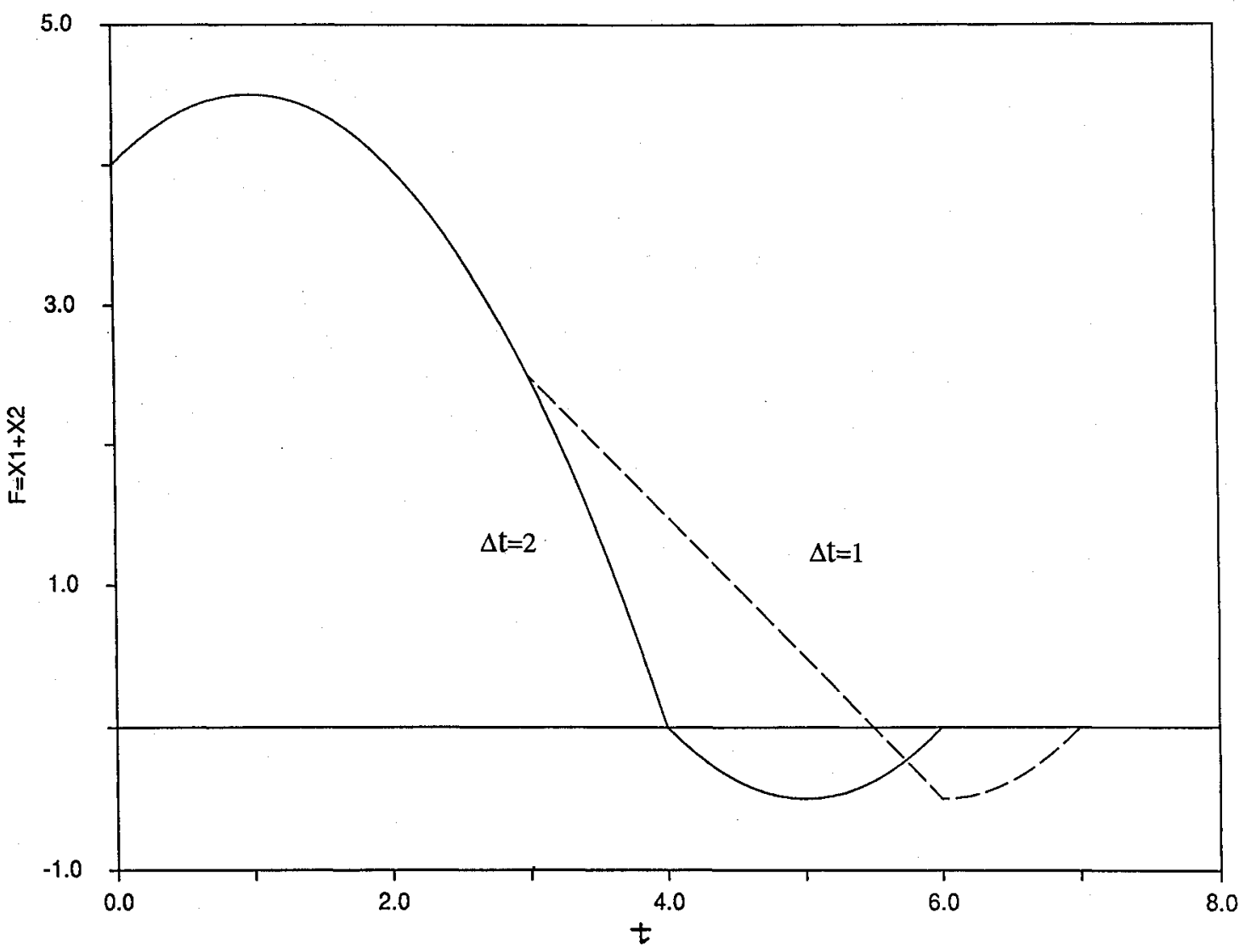

Fig. 1 Variation of $F=x_{1}+x_{2}$. 


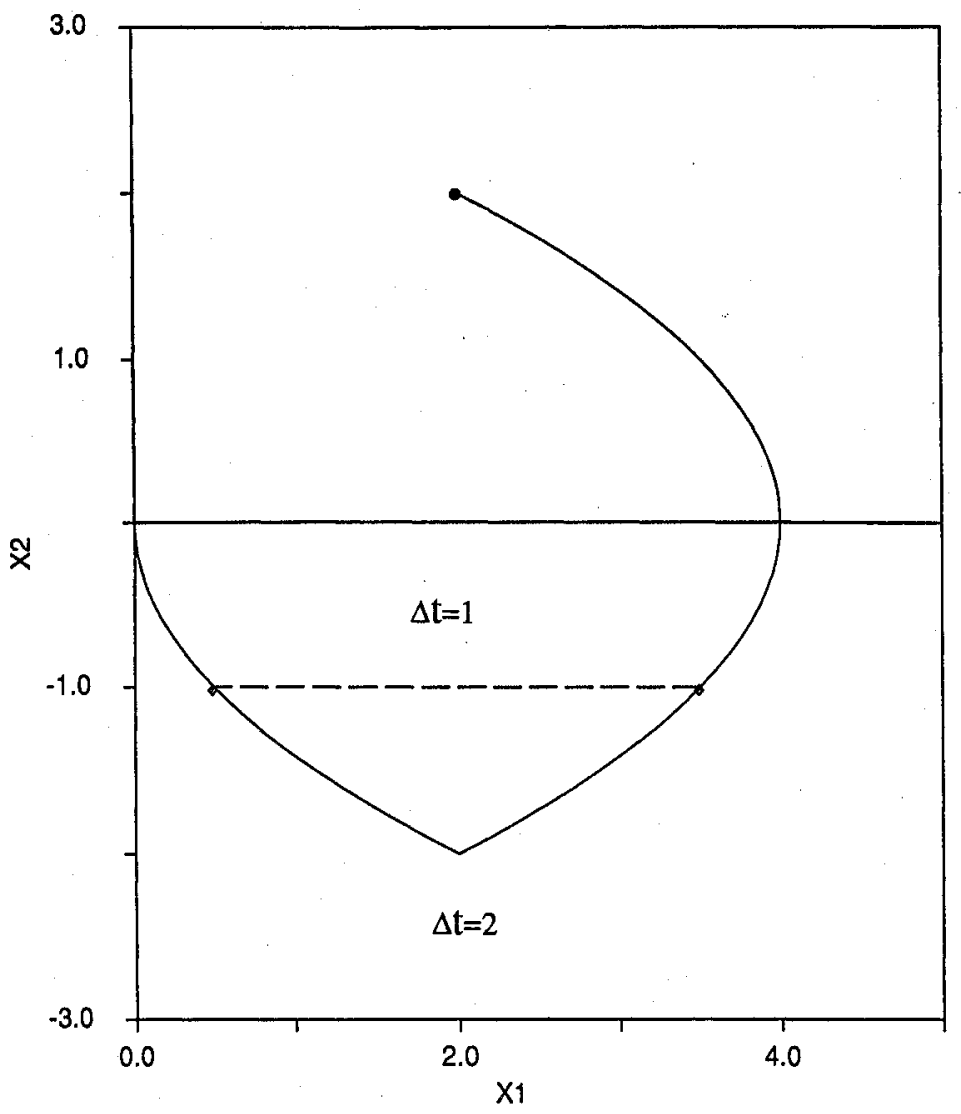

Fig. 2 Trajectory in the phase plane.

direction as long as the distance $d$ is the same. Then concern may be focused on the highest risk encountered, i.e., the risk cost function can be formed as

$$
J_{r}=\max _{t_{0} \leq t \leq t_{f}} C_{1} \exp \left[-C_{2} d(t)\right]
$$

Or, more directly, the minimum distance along the trajectory from the threat can be used as a measure of the highest risk

$$
J_{r}=\min _{t_{0} \leq t \leq t_{f}} d^{2}(t)=\max _{t_{0} \leq t \leq t_{f}}-d^{2}(t)
$$

The goal is to minimize an overall cost function:

$$
\min J=\min \left\{\max _{t_{0} \leq t \leq t_{f}} F(x(t))+\psi\left(x\left(t_{f}\right), t_{f}\right)\right\}
$$

where $F(x(t))$ can be either the exponential function in Eq. (74) or $-d^{2}(t)$, as in Eq. (75). Now the problem becomes an optimal control problem with maximum functional. We shall apply the necessary conditions stated in Sec. II to solve the problem.

Let us consider a nonthrusting subsonic aeroglider, flying from point $P_{1}$ to point $P_{2}$ in a horizontal plane of certain altitude. A flat Earth is assumed. The initial and final speeds of the vehicle are specified. Assuming that the vehicle has a parabolic drag polar of the form

$$
C_{D}=C_{D 0}+K C_{L}^{2}
$$

we have the nondimensional equations of motion for the vehicle ${ }^{21}$ :

$$
\begin{aligned}
& \frac{\mathrm{d} x}{\mathrm{~d} \tau}=v \cos \psi \\
& \frac{\mathrm{d} y}{\mathrm{~d} \tau}=v \sin \psi
\end{aligned}
$$

$$
\begin{gathered}
\frac{\mathrm{d} v}{\mathrm{~d} \tau}=-\left(\frac{v^{2}}{2 E^{*} \omega}\right)\left(1+\frac{\omega^{2}}{v^{4} \cos ^{2} \sigma}\right) \\
\frac{\mathrm{d} \psi}{\mathrm{d} \tau}=\frac{\tan \sigma}{v}
\end{gathered}
$$

where all variables are dimensionless, with the definitions given in Ref. $21 ; x$ and $y$ are the position coordinates, $v$ is the speed and $\psi$ is the heading angle, the control is the bank angle $\sigma$, and $E^{*}$ is the maximum lift-to-drag ratio. The constant $\omega$ depends on the vehicle characteristics and the altitude. To maintain the horizontal flight, the equilibrium condition

$$
L / W=1 / \cos \sigma
$$

must hold, where $L$ is the lift and $W$ is the weight. A constraint on the load factor $n=L / W \leq n_{\max }=3$ is imposed. As a result of Eq. (82) and $n \leq n_{\max }$, an effective constraint on $\sigma$ is needed ${ }^{21}$ :

$$
|\sigma| \leq \sigma_{\max }=\min \left\{\cos ^{-1}(1 / 3), \cos ^{-1}\left(\omega C_{L \max } / \nu^{2} C_{L}^{*}\right)\right\}
$$

where $C_{L \max }$ is the maximum lift coefficient and $C_{L}^{*}$ is the lift coefficient corresponding to the maximum lift-to-drag ratio. Suppose that the coordinate system is set up such that a threat, e.g., a thunderstorm, is centered at the origin, and the flight is to meet the following mission requirements:

$$
\begin{aligned}
& x_{0}=-1.5, \quad y_{0}=0, \quad \psi_{0}=0, \quad v_{0}=1 \\
& x_{f}=3, \quad y_{f}=0, \quad \psi_{f}=\text { free }, \quad v_{f}=v_{\text {stall }}
\end{aligned}
$$

The final speed $v_{\text {stall }}$ is the stall speed. For computation, we take

$$
\begin{gathered}
\omega=0.23, \quad C_{L \max } / C_{L}^{*}=1.8, \quad E^{*}=20 \\
v_{\text {stall }}=\sqrt{\omega C_{L}^{*} / C_{L \max }}=0.3575
\end{gathered}
$$


We define the risk cost as the highest probability of being hit by lightning, which is directly proportional to the minimum distance from the center of the thunderstorm. The objective is to reduce the exposure to the threat and to prolong the flight endurance. So the cost function

$$
J=\left[\max _{0 \leq \tau \leq \tau_{f^{\prime}}}-\left(x^{2}+y^{2}\right)-\tau_{f}\right]
$$

is to be minimized. To apply the necessary conditions, we first form the Hamiltonian

$$
\begin{aligned}
H & =p_{x} v \cos \psi+p_{y} v \sin \psi-p_{v} \frac{v^{2}}{2 E^{*} \omega}\left(1+\frac{\omega^{2}}{v^{4} \cos ^{2} \sigma}\right) \\
& +p_{\psi} \frac{\tan \sigma}{v}=-1
\end{aligned}
$$

After writing down the adjoint system (19), it is easy to show that three first integrals exist:

$$
\begin{gathered}
p_{x}=c_{1} \\
p_{y}=c_{2} \\
p_{\psi}=c_{1} y-c_{2} x+c_{3}
\end{gathered}
$$

where $c_{i}$ is constant. The optimality condition (21) gives the control

$$
u=\tan \sigma= \begin{cases}p_{\psi} E^{*} v / p_{v} \omega, & \text { if }|\sigma| \leq \sigma_{\max } \\ \tan \sigma_{\max } \operatorname{sign}\left(p_{\psi}\right), & \text { otherwise }\end{cases}
$$

The second equation in (91) uses the fact that $p_{v}>0$, required by the second-order condition $\partial^{2} H / \partial u^{2}>0$. When $|\sigma| \leq \sigma_{\max }$, substituting Eqs. (88-91) into Eq. (87), and defining

$$
A=c_{1} y-c_{2} x+c_{3}
$$

$$
\begin{gathered}
B=2 v\left[1+v\left(c_{1} \cos \psi+c_{2} \sin \psi\right)\right] \\
C=-A\left(\omega^{2}+v^{4}\right) / \omega^{2}
\end{gathered}
$$

we can solve for $\tan \sigma$ to obtain the control law

$$
\tan \sigma=\frac{-B+\sqrt{B^{2}-4 A C}}{2 A}
$$

If $-\left(x^{2}+y^{2}\right)$ attains an isolated maximum only once at $\tau_{1} \in\left(0, \tau_{f}\right)$, the jump conditions by Eqs. (30) and (31) are

$$
\begin{aligned}
& c_{1}^{+}=c_{1}^{-}-2 x_{1} \\
& c_{2}^{+}=c_{2}^{-}-2 y_{1}
\end{aligned}
$$

Note that $p_{\psi}$ and $p_{v}$ are not involved in the jump conditions and therefore are continuous at $\tau_{1}$. Note, also, that substituting Eqs. (96) and (97) into Eq. (90) verifies that $p_{\psi}^{+}=p_{\psi}^{-}$. Since $F=-\left(x^{2}+y^{2}\right)$ is of second order by Definition 2 of Sec. II, if $F$ has a flat maximum in $\left(\tau_{1}, \tau_{2}\right)$, Necessary Condition III applies. The control in $\left(\tau_{1}, \tau_{2}\right)$ is given by setting $\ddot{F}(x, y)=0$ :

$$
\tan \sigma=\frac{v^{2}}{x \sin \psi-y \cos \psi}
$$

All the necessary conditions constitute an equivalence of a nonlinear root-finding problem. In general, a homotopy method may be used to solve for the solution. ${ }^{13}$ But in this particular application, the nonlinear programming approach

Table 1 Summary of results

\begin{tabular}{lcll}
\hline \multicolumn{1}{c}{ Trajectory type } & Flight endurance $\tau_{f}$ & $d_{\min }$ & $d_{\min }^{2}+\tau_{f}$ \\
\hline Threat avoidance & 9.62 & 1.17 & 10.989 \\
Minimax & 9.42 & 1.2 & 10.86 \\
Maximum endurance & 9.73 & 0.52 & 10.011 \\
\hline \hline
\end{tabular}

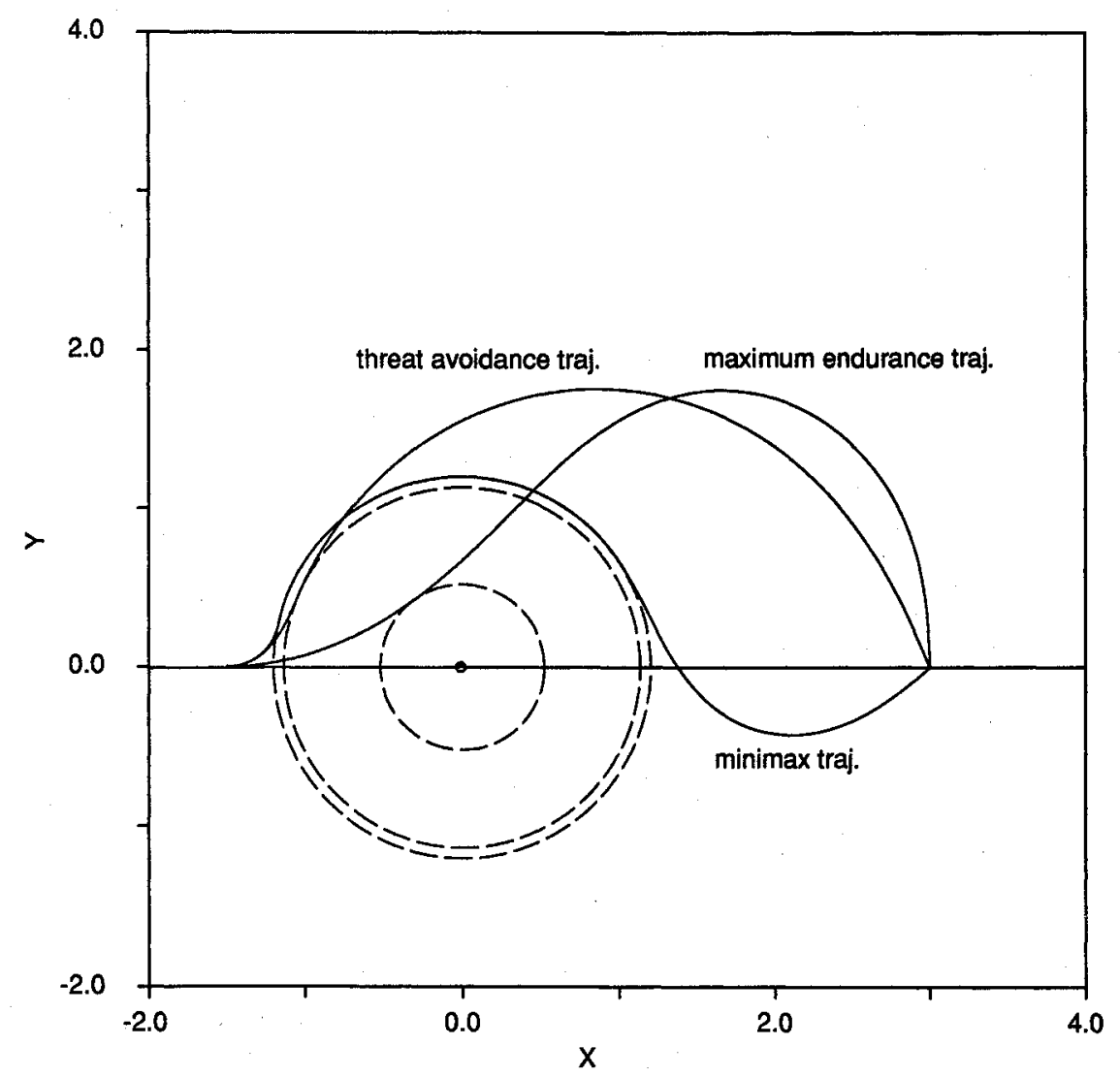

Fig. 3 Glider trajectories in the $x-y$ plane. 


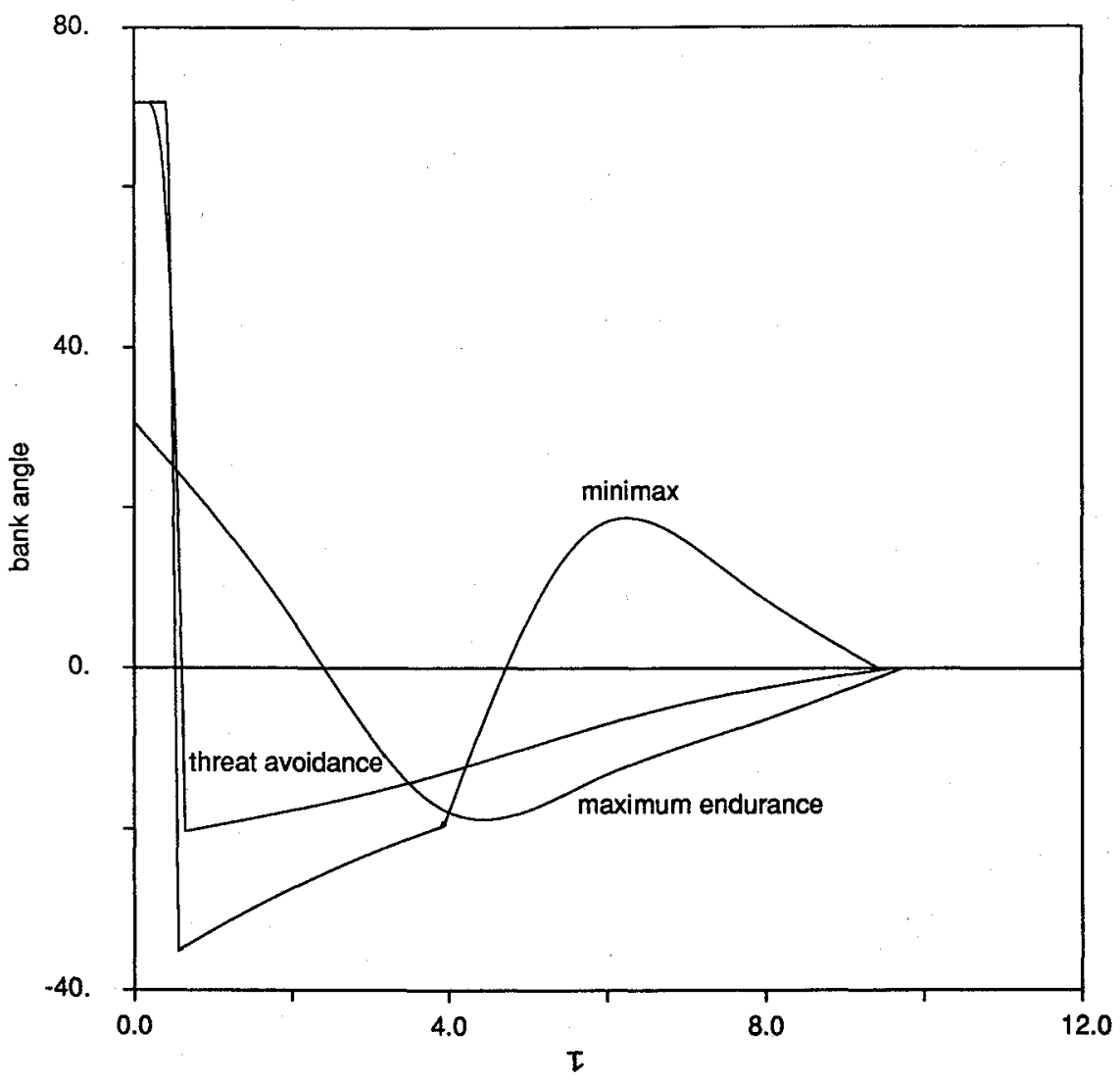

Fig. 4 Histories of bank angle $\sigma$ (deg).

works reasonably well. A quasi-Newton algorithm ${ }^{22}$ is employed for this purpose.

For comparison, the minimax problem with $J=\max \left(-d^{2}\right)$ and the maximum endurance problem with $J=-\tau_{f}$ are also solved. The results are listed in Table 1 . It was found that the given condition $d(t)$ has only an isolated maximum, occurring at $\tau_{1}=0.64$, for the threat avoidance problem. The optimal threat avoidance solution indeed increases the minimum distance from the threat by a factor of more than two without severe reduction in the flight time, as compared with the maximum endurance solution. The reason for the isolated maximum instead of flat maximum is that Eq. (98) tends to generate a larger bank angle than Eq. (95) does, hence it is not favorable for a prolonged flight endurance. If the flight time is not a concern, a flat maximum is likely to occur. This is confirmed by the minimax solution. For the minimax problem, all the necessary conditions apply, except for $H=0$, as opposed to Eq. (87). In this case, $d^{2}=\left(x^{2}+y^{2}\right)$ has a flat maximum $d_{\min }=1.2$, with a flight time of $\tau_{f}=9.42$. Notice that among three trajectories the threat avoidance solution provides the maximum value of $d_{\min }^{2}+\tau_{f}$. Figure 3 shows three trajectories in the $x-y$ plane. The corresponding bank angle histories are in Fig. 4. For both the threat avoidance and minimax solutions, the bank angle starts with the maximum magnitude for a tight turn away from the threat. Also note that the bank angle is continuous everywhere for both cases, but has discontinuous slope, due to the jump conditions and departure from the boundary arc.

\section{Conclusions}

This paper considers the optimal control problem with a performance index that includes a maximum functional. The problem is transformed into a problem of Mayer, with inequality state constraint using a well-known technique. A set of necessary conditions is derived. The results obtained beyond earlier work mainly result from the fact that less stringent assumptions are placed on the problem and multiple maxima are allowed to occur; therefore, a broader class of problems can be treated. In addition, some previous work is proved to be reducible and thus included in our results.

The application in aerospace trajectory optimization is demonstrated by formulating the threat avoidance problem within the context of the type of problems discussed in this paper. The necessary conditions are followed to solve the problem for a subsonic glider. Numerical results are presented.

\section{Appendix: Proof of Proposition 1 in Section III}

We need to show that $\lambda=0$ in Eq. (49). In the more detailed work of the calculus of variations, ${ }^{23,24}$ it has been shown in the imbedding lemmas that a nontangence condition for the constraint (46) is required for a normality property. The normality in turn is required for the existence of a one-parameter family of solution $x(t, \epsilon)$ and $u(t, \epsilon)$, satisfying state equation (45) and the intermediate point constraint (46), and, more importantly, for Eq. (49) to be valid. This nontangence condition in this case is that

$$
\begin{aligned}
& \dot{F}_{x}^{T}\left(x^{*}\left(t_{1}\right)\right) \dot{x}^{*}\left(t_{1}^{+}\right) \neq 0 \\
& \dot{F}_{x}^{T}\left(x^{*}\left(t_{1}\right)\right) \dot{x}^{*}\left(t_{1}^{-}\right) \neq 0
\end{aligned}
$$

where we distinguish the left and right limit values of functions at $t_{1}$ by $\left(^{-}\right)$and $\left(^{+}\right)$signs when necessary, because we do not exclude the possibility of discontinuous $u^{*}(t)$ at $t_{1}$. Since $\dot{F}(x(t))$ does not contain $u$ explicitly, Eqs. (A1) and (A2) really amount to

$$
\begin{aligned}
& \ddot{F}\left(t_{1}^{+}\right) \neq 0 \\
& \ddot{F}\left(t_{1}^{-}\right) \neq 0
\end{aligned}
$$

Since $F\left(x *\left(t_{1}\right)\right)$ is the maximum of $F\left(x^{*}(t)\right), \ddot{F}\left(t_{1}^{+}\right)$and $\ddot{F}\left(t_{1}^{-}\right)$must be nonpositive. According to Eqs. (A3) and (A4), they should be strictly negative:

$$
\ddot{F}\left(t_{1}^{+}\right)<0
$$




$$
\ddot{F}\left(t_{1}^{-}\right)<0
$$

From the Weierstrass condition, ${ }^{4}$ we know that for any $\boldsymbol{u} \in U$ and any $t \in\left(t_{0}, t_{f}\right)$,

$$
H\left(x^{*}(t), p(t), u^{*}(t)\right) \geq H\left(x^{*}(t), p(t), u\right)
$$

From the fact that $H\left(x^{*}(t), p(t), u^{*}(t)\right)$ is continuous throughout $\left(t_{0}, t_{f}\right)$, we have

$$
\boldsymbol{p}^{T}\left(t_{1}^{-}\right) f\left(x^{*}\left(t_{1}\right), u^{*}\left(t_{1}^{-}\right)\right)=p^{T}\left(t_{1}^{+}\right) f\left(x^{*}\left(t_{1}\right), u^{*}\left(t_{1}^{+}\right)\right)
$$

Replacing $p\left(t_{1}^{+}\right)$by Eq. (49) in Eq. (A8) gives

$$
\begin{aligned}
& p^{T}\left(t_{1}^{-}\right) f\left(x^{*}\left(t_{1}\right), u^{*}\left(t_{1}^{-}\right)\right)=p^{T}\left(t_{1}^{-}\right) f\left(x^{*}\left(t_{1}\right), u^{*}\left(t_{1}^{+}\right)\right) \\
& \quad+F_{x}^{T}\left(x^{*}\left(t_{1}\right)\right) f\left(x^{*}\left(t_{1}\right), u^{*}\left(t_{1}^{+}\right)\right) \\
& \quad+\lambda \dot{F}_{x}^{T}\left(x^{*}\left(t_{1}\right)\right) f\left(x^{*}\left(t_{1}\right), u^{*}\left(t_{1}^{+}\right)\right)
\end{aligned}
$$

Notice that

$$
\begin{gathered}
F_{x}^{T}\left(x^{*}\left(t_{1}\right)\right) f\left(x^{*}\left(t_{1}\right), u^{*}\left(t_{1}^{+}\right)\right)=\dot{F}\left(x^{*}\left(t_{1}\right)\right)=0 \\
\dot{F}_{x}^{T}\left(x^{*}\left(t_{1}\right)\right) f\left(x^{*}\left(t_{1}\right), u^{*}\left(t_{1}^{+}\right)\right)=\ddot{F}\left(t_{1}^{+}\right)
\end{gathered}
$$

Equation (A9) then leads to

$$
\begin{aligned}
& H\left(\boldsymbol{x}^{*}\left(t_{1}\right), \boldsymbol{p}\left(t_{1}^{-}\right), u^{*}\left(t_{1}^{-}\right)\right) \\
& \quad-H\left(\boldsymbol{x}^{*}\left(t_{1}\right), \boldsymbol{p}\left(t_{1}^{-}\right), u^{*}\left(t_{1}^{+}\right)\right)=\lambda \ddot{F}\left(t_{1}^{+}\right)
\end{aligned}
$$

Let $\boldsymbol{u}=\boldsymbol{u}^{*}\left(t_{1}^{+}\right)$in Eq. (A12); Eq. (A7) indicates that

$$
\lambda \ddot{F}\left(t_{1}^{+}\right) \geq 0
$$

From Eqs. (A5) and (A13), we have

$$
\lambda \leq 0
$$

Likewise, using Eq. (49) to replace $p\left(t_{1}^{-}\right)$in Eq. (A8) yields

$$
\begin{aligned}
& H\left[x^{*}\left(t_{1}\right), p\left(t_{1}^{+}\right), u^{*}\left(t_{1}^{+}\right)\right. \\
& \quad-H\left[x^{*}\left(t_{1}\right), p\left(t_{1}^{+}\right), u^{*}\left(t_{1}^{-}\right)\right]=-\lambda \ddot{F}\left(t_{1}^{-}\right)
\end{aligned}
$$

Hence, by Eq. (A7), we have

$$
-\lambda \ddot{F}\left(t_{1}^{-}\right) \geq 0
$$

Combining Eqs. (A6) and (A16) gives

$$
\lambda \geq 0
$$

Eqs. (A14) and (A17) imply

$$
\lambda=0
$$

\section{References}

'Johnson, C. D., "Optimal Control Problem with Chebyshev Minimax Performance Index," Journal of Basic Engineering, Vol. 89, 1967, pp. 251-262.

${ }^{2}$ Barry, P. E., "Optimal Control with Minimax Cost," IEEE Trans- actions on Automatic Control, Vol. AC-16, Aug. 1971, pp. 354-357.

${ }^{3}$ Neustadt, L. W., Optimization: A Theory of Necessary Conditions, Princeton Univ. Press, Princeton, NJ, 1976.

${ }^{4}$ Lee, E. B., and Markus, L., Foundations of Optimal Control Theory, Wiley, New York, 1967.

${ }^{5}$ Powers, W. F., "A Chebyshev Minimax Technique Oriented to Aerospace Trajectory Optimization Problems," AIAA Journal, Vol. 10, 1972, pp. 1291-1296.

${ }^{6}$ Miele, A., and Venkataraman, P., "Optimal Trajectories for Aeroassisted Orbital Transfer," Acta Astronautica, Vol. 11, Nos. 7-8, 1984, pp. 423-433.

${ }^{7}$ Miele, A., and Basapur, V. K., "Approximate Solution to Minimax Optimal Control Problems for Aeroassisted Orbital Transfer," Acta Astronautica, Vol. 12, No. 10, 1985, pp. 809-818.

${ }^{8}$ Miele, A., Mohanty, B. P., Venkataraman, P., and Kuo, Y. M., "Numerical Solution of Minimax Problems of Optimal Control, Part 1," Journal of Optimization Theory and Applications, Vol. 38, No. 1, 1982, pp. 97-109.

${ }^{9}$ Miele, A., Basapur, V. K., and Lee, M. Y., "Optimal Trajectories for Aeroassisted, Coplanar Orbital Transfer," Journal of Optimization Theory and Applications, Vol. 52, No. 1, 1987, pp. 1-24.

${ }^{10}$ Miele, A., Wang, T., and Melvin, W. W., "Optimization and Acceleration Guidance of Flight Trajectories in a Windshear," Journal of Guidance, Control, and Dynamics, Vol. 10, No. 4, 1989, pp. 368-377.

${ }^{11}$ Miele, A., Wang, T., Tzeng, C. Y., and Melvin, W. W., "'Optimal Abort Landing Trajectories in the Presence of Windshear," Journal of Optimization Theory and Applications, Vol. 55, No. 2, 1987, pp. 165-202.

${ }^{12}$ Miele, A., Wang, T., Melvin, W. W., and Bowles, R. L., “Acceleration, Gamma, and Theta Guidance for Abort Landing in a Windshear," Journal of Guidance, Control, and Dynamics, Vol. 12, No. 6, 1989 , pp. 815-821.

${ }^{13}$ Oberle, H. J., "Numerical Treatment of Minimax Optimal Control Problems with Application to Reentry Flight Path Problem,", Journal of the Astronautical Sciences, Vol. 36, Nos. 1-2, 1988, pp. 159-178.

${ }^{14} \mathrm{Lu}$, P., and Vinh, N. X., "Minimax Optimal Control for Atmospheric Fly-Through Trajectories," Journal of Optimization Theory and Applications, Vol. 57, No. 1, 1988, pp. 41-58.

${ }^{15}$ Vinh, N. X., and Lu, P., "Chebyshev Minimax Problems for Skip Trajectories," Journal of the Astronautical Sciences, Vol. 36, Nos. 1-2, 1988, pp. 179-197.

${ }^{16} \mathrm{Lu}$, P., "Minimax and Maximax Optimal Control Problems with Applications in Aerospace Engineering," Ph.D. Dissertation, Univ. of Michigan, Ann Arbor, MI, April 1988.

${ }^{17}$ Pontryagin, L. S., Boltyanskii, V. G., Gramkreledze, Q. V., and Mishchenko, E. F., The Mathematical Theory of Optimal Processes, Interscience, New York, 1962, Chap. 6.

${ }^{18}$ Bryson, A. E., and Ho, Y. C., Applied Optimal Control: Optimization, Estimation, and Control, Hemisphere, New York, 1975, Chap. 3.

${ }^{19}$ Warga, J., "Minimax Problems and Unilateral Curves in the Calculus of Variations," SIAM Transactions on Control, Vol. 3, No. 1, 1965, pp. 91-105.

${ }^{20}$ Vian, J. L., and Moore, J. R., "Trajectory Optimization with Risk Minimization For Military Aircraft," Journal of Guidance, Control, and Dynamics, Vol. 12, No. 3, 1989, pp. 311-317.

${ }^{21}$ Vinh, N. X., Yang, Y. C., and Chern, J. S., "Optimal Trajectories for Maximum Endurance Gliding in a Horizontal Plane," Journal of Guidance, Control, and Dynamics, Vol. 7, No. 2, 1984, pp. 246-248.

${ }^{22}$ Shanno, D. F., and Phua, H. K., "Minimization of Unconstrained Multivariate Functions," ACM Transactions on Mathematical Software, Vol. 6, No. 6, 1980, pp. 618-622.

${ }^{23}$ Bliss, G. A., Lectures on the Calculus of Variations, Univ. of Chicago Press, Chicago, IL, 1946.

${ }^{24}$ Troitskii, V. A., "Variational Problems of Control Processes with Functionals Depending Upon the Intermediate Values of the Coordinates," PMM, Vol. XXVI, No. 6, 1962. 